\title{
La violence en temps de guerre et en temps de paix.
} Leçons de l'après-guerre froide : l'exemple du Salvador. Partie 1

Philippe Bourgois

\section{(2) OpenEdition \\ Journals}

Édition électronique

URL : http://journals.openedition.org/conflits/825

DOI : $10.4000 /$ conflits. 825

ISSN : $1777-5345$

Éditeur :

CCLS - Centre d'études sur les conflits lilberté et sécurité, L'Harmattan

\section{Édition imprimée}

Date de publication : 1 septembre 2002

ISBN : 2-7475-3432-4

ISSN : $1157-996 \mathrm{X}$

\section{Référence électronique}

Philippe Bourgois, « La violence en temps de guerre et en temps de paix. Leçons de l'après-guerre froide : I'exemple du Salvador. Partie $1 »$, Cultures \& Conflits [En ligne], 47 | automne 2002, mis en ligne le 29 avril 2003, consulté le 30 mars 2021. URL : http://journals.openedition.org/conflits/825 ; DOI : https://doi.org/10.4000/conflits.825

Ce document a été généré automatiquement le 30 mars 2021.

Creative Commons License 


\title{
La violence en temps de guerre et en temps de paix. Leçons de l'après- guerre froide : l'exemple du Salvador. Partie 1
}

\author{
Philippe Bourgois
}

La violence en temps de guerre et en temps de paix

Leçons de l'après-guerre froide : l'exemple du Salvador ${ }^{1}$

"Quand les bombardements et les mitraillages au sol ont commencé, on m'a ordonné de m'accroupir derrière un tronc d'arbre, et quoiqu'il arrive, de rester immobile. Ils tiraient sur tout ce qui bougeait.

Durant les quatre premiers jours, une quinzaine de femmes et d'enfants ont été blessés, des éclats d'obus ont été retirés et des amputations pratiquées sans aucun anesthésiant. Les troupes gouvernementales qui nous encerclaient se rapprochaient et les combats montaient en intensité, tuant tous ceux qui se trouvaient sur leur chemin.

A la quatrième nuit, alors que nous courions sur un chemin accidenté, nous avons atteint la ligne de feu des troupes gouvernementales. Les bébés que les femmes portaient se mirent à crier au bruit des tirs et dès que nous nous sommes trouvés à portée de voix des troupes gouvernementales, celles-ci ont dirigé leurs tirs droit sur nous.

C'était un véritable enfer, des grenades explosaient tout autour, les mitrailleuses crachaient, nous courions, trébuchions, tombions, tentant de traverser le barrage de balles et d'obus. A vingt pas devant moi, un petit garçon hors d'haleine, a été touché par une grenade. Son corps gisait au milieu du sentier étroit. J'ai dû courir droit sur lui pour m'échapper ».

Washington Post, 28 décembre 1982.

«Durant les treize premiers mois que j'ai passés dans Spanish Harlem, j'ai été le témoin : 
- d'une fusillade mortelle, devant ma fenêtre, par un tireur brandissant une carabine à canon scié, tuant la mère d'un petit garçon de trois ans,

- d'une explosion et d'une rafale de mitrailleuse encore une fois visibles depuis ma fenêtre,

- d'un tir et d'une course poursuite par la police devant une pizzeria, alors que j'étais en train d'y manger,

- de la fin d'un incendie, par cocktail Molotov, d'unecrack house,

- d'une douzaine de bagarres de rues (cris, vêtements déchirés),

sans compter les rencontres quotidiennes avec des êtres humains anéantis, certains en proie à des paranoïas dues à l'absorption de crack, d'autres victimes de delirium tremens, d'autres encore souffrant de pathologies non identifiées et lançant des insultes à tout ce qui les entourait.

La prégnance d'une culture de terreur a peut-être trouvé son expression la plus poignante dans un commentaire que m'a fait un garçon de treize ans au cours d'une conversation anodine à propos de l'état d'évolution de la grossesse de sa maman. Il m'a dit qu'il espérait que le bébé serait un garçon «parce que les filles, c'est trop facile à violer $»$.

New York Times Magazine, 12 novembre 1989.

Ces citations sont extraites d'articles de journaux que j'ai écrits dans les années 1980 afin d'attirer l'attention sur la violence sur deux de mes terrains d'enquête. La première recherche était menée parmi les paysans révolutionnaires du Salvador, la deuxième portait sur la deuxième génération de dealers de crack portoricains à East Harlem, dans la ville de New York. En passant d'un site à un autre, je me suis intéressé à la différenciation des formes de la violence et des significations qui lui sont données en temps de guerre et en temps de paix, afin d'illustrer les façons dont elle met en question, ou renforce au contraire, les inégalités de pouvoir. Sur la scène révolutionnaire du Salvador, je voulais documenter la capacité réelle des dominés à résister à la répression d'Etat; aux Etats-Unis, je me battais pour expliquer l'effet de démobilisation politique, fruit des conflits interpersonnels et l'autodestruction qui se propagent dans la ville de New York. Plus d'une décennie plus tard, encouragé par l'extension de la dérégulation du capitalisme à l'échelle mondiale, je reviens sur ces comptes-rendus de la violence des années 1980 et les confronte à de nouvelles observations ethnographiques qui me font penser que mes descriptions empiriques et mes analyses théoriques d'alors étaient profondément affectées par le contexte politique. En Amérique centrale, j'ai travaillé en étant inconsciemment soumis aux contraintes de la Guerre froide, qui m'ont conduit à édulcorer mes descriptions de la violence politique chez les paysans révolutionnaires. Sur le plan théorique, cela a obscurci la vision du caractère multiple des manifestations de violence et la standardisation de ces différents sous-types de violence au travers des prismes historique, culturel et politique. Endosser les «lunettes» de la guerre froide m'a conduit à méconnaître le pouvoir de la violence qui conforte les cadres d'inégalité 
sociale aux yeux du public, et à dépolitiser les tentatives de résistance à l'oppression dans un Salvador en guerre. Par contraste, dans les situations urbaines faisant intervenir des enjeux raciaux aux Etats-Unis, j'étais en mesure de critiquer les effets de démobilisation de la violence quotidienne en montrant comment elle résulte de l'intériorisation d'une violence structurale profondément ancrée chez les acteurs et comment elle s'exprime dans un maelström d'agressions banalisées, interpersonnelles et délinquantes.

Pour avoir une compréhension plus complexe du Salvador révolutionnaire et du déclin des villes américaines, il me semble utile de distinguer entre quatre types de violence : politique, structurale, symbolique et quotidienne (voir encadré en fin d'article). Le terme de "violence politique " désigne une répression physique, directement et intentionnellement exercée par l'armée ou la police à l'encontre d'opposants, au nom d'une idéologie, d'un mouvement politique ou d'un Etat - ou son contraire : un combat armé populaire contre un régime répressif. Le terme de "violence structurale " se réfère à l'organisation politico-économique de la société qui impose des conditions de détresse physique et psychologique, allant des taux élevés de mortalité aux conditions de travail abusives. Cette violence est enracinée, à une échelle macro, dans des structures: le caractère inégal du commerce international, par exemple, s'exprime localement par une forte exploitation des salariés, le truquage des marchés et le monopole des services. Le terme a d'abord été défini dans les cercles universitaires par le fondateur des Peace and Conflict Studies, Johan Galtung', pour insister sur l'engagement de la social-démocratie en faveur des droits de l'Homme $^{3}$ et pour repousser l'hystérie anti-communiste propagée par le capitalisme nord-américain durant la guerre froide, sensible dans la répression politique de la contestation populaire dans le monde non-industrialisé. Des racines radicales de la violence structurale se retrouvent aussi dans les mouvements de résistance anti-coloniaux ${ }^{4}$ et dans les revendications de la théologie de la libération en faveur d'un «choix préférentiel pour les pauvres $»^{5}$. Plus récemment, le concept a été utilisé par les anthropologues de la médecine pour analyser la façon dont les inégalités économiques extrêmes favorisent maladie et souffrance sociale ${ }^{6}$. Le concept de "violence symbolique» a été développé par Pierre Bourdieu pour montrer comment la domination opère dans des dimensions intimes de la vie des agents sociaux. On pense par exemple à la méconnaissance des structures de pouvoir par les dominés qui participent à leur propre oppression chaque fois qu'ils perçoivent et jugent l'ordre social à travers des catégories qui rendent cette oppression naturelle et évidente ${ }^{7}$.

Le concept de "violence quotidienne » a enfin été développé de façon extrêmement parlante par N. Scheper-Hughes ${ }^{8}$ pour attirer notre attention sur une dimension plus phénoménologique des "crimes en temps de paix », sur les "petites guerres » et les "génocides invisibles » qui frappent les dominés. L'usage que N. Scheper-Hughes fait de ce mot tend à assimiler la violence quotidienne à la violence structurale ou institutionnelle. Je crois plus approprié de limiter la notion aux pratiques routinières et aux expressions ordinaires d'agression interpersonnelle qui servent à normaliser la violence, à une échelle micro, dans les conflits familiaux ou sexuels, dans les actes de délinquance ou dans la consommation de drogues. L'importation analytique du mot permet d'éviter de donner une explication psychologique ou individualiste aux confrontations interpersonnelles. Ma définition la plus étroite est appropriée pour dépeindre comment la violence quotidienne peut croître et cristalliser une « culture de terreur ", pour citer Taussig ${ }^{9}$, qui établit un sens commun de la violence et en fait une 
évidence allant de soi tant dans les sphères publiques que privées. Dans cet article, la ré-interprétation de mes données ethnographiques montrera comment j'ai été incapable de reconnaître, au Salvador, le caractère spécifique de la violence quotidienne, donc de la percevoir et de l'analyser comme produit de la violence politique et structurale - alors même que je l'avais identifiée dans les villes nordaméricaines, à l'interface de la violence symbolique et de la violence structurale.

La politique de la représentation au Salvador durant la guerre froide

La première citation en exergue dépeint l'éradication militaire des paysans révolutionnaires au Salvador. Elle a été écrite en 1981 au moment de l'escalade finale de la guerre froide. Le Salvador était en proie à une guerre civile opposant un gouvernement militaire de droite à une coalition d'organisations de gauche pratiquant la guérilla et plus connue sous le nom de Front Farabundo Martí de Libération Nationale (FMLN). Pendant presque tout le XXe siècle, les Etats-Unis ont invoqué la rhétorique de la défense du monde libre contre le communisme pour justifier leur soutien à une série de régimes militaires dans le pays. Ces gouvernements ont promu les intérêts politiques et économiques d'une petite oligarchie du café plus connue sous le nom des "quatorze familles » et très remarquée pour ses violations systématiques des droits de l'Homme. Près de 75000 Salvadoriens, principalement des civils, ont péri dans les années 1980, dans la répression par l'Etat des guérillas FMLN et de leurs sympathisants. Au moment où je menais mes recherches sur le terrain, 800 personnes en moyenne étaient tuées chaque mois par les militaires salvadoriens et les escadrons de la mort qui leur étaient affiliés ${ }^{10}$. Durant cette période, le gouvernement dépendait pour sa propre survie du soutien militaire, politique et économique des Etats-Unis, recevant au total plus de 4 milliards de dollars durant les années 1980, bien plus que tout autre pays, Egypte et Israël exceptés ${ }^{11}$.

Cette présentation de la situation salvadorienne était fondée sur un projet de thèse avorté se proposant d'examiner la mobilisation des paysans salvadoriens durant la guerre civile dans chacun des deux camps. Pour conduire cette recherche, je me suis rendu dans une région rurale frappée par la guerre où presque toute la population soutenait activement les combattants de la guérilla du FMLN. Deux jours après mon arrivée, je me suis retrouvé pris, avec les villageois, dans les opérations de terre brûlée menées par le gouvernement. Des troupes régulières encerclaient une région d'une vingtaine de $\mathrm{km}$ » qui comprenait une douzaine de petits villages de paysans soutenant le FMLN et qui était la cible de bombardements aériens. Les militaires ont poursuivi leurs actions avec l'infanterie, en détruisant le plus d'infrastructures possible (récoltes, bétail, habitations) et en tuant, parfois en torturant, les gens qu'ils capturaient. Avec la population civile, soit près de mille paysans, j'ai couru quatorze jours durant pour sauver ma peau avant de me retrouver en sécurité en tant que réfugié au Honduras voisin. Accompagnés par à peine plus d'une centaine de combattants armés du FMLN, nous nous cachions le jour et fuyions la nuit. Les guérilleros, dont la plupart étaient nés et avaient grandi dans la région, se déplaçaient sur nos flancs afin de tenter de nous protéger, mais nous étions sans cesse mitraillés, bombardés et poursuivis par les avions, les hélicoptères et les troupes au sol de l'armée salvadorienne. Les soldats gouvernementaux étaient guidés par des combattants paramilitaires particulièrement brutaux et recrutés dans les villages voisins ${ }^{12}$.

A l'époque, il me semblait que la répression d'Etat à l'encontre des populations civiles échouait. Je pensais que la douleur, la peur et l'angoisse engendrées par la campagne 
militaire renforçaient l'engagement idéologique et émotionnel de la population civile dans le sens de la rébellion, bref, que la répression radicalisait les petits agriculteurs marginalisés. J'interprétais la mobilisation de ces derniers dans la lutte armée comme une libération sociale autant qu'individuelle, un peu comme Franz Fanon et Jean-Paul Sartre ${ }^{13}$ avaient célébré la guerre anti-coloniale des Algériens contre la France. Les paysans salvadoriens s'organisaient alors autour d'une idéologie syncrétique qui mêlait la théologie catholique de la libération, une conception marxiste de la lutte des classes, un populisme socialiste romantique et un sens de la vengeance sociale et de la dignité personnelle ${ }^{14}$. Bien plus important à mes yeux était alors leur rejet quasi-messianique de l'humiliation et de l'exploitation par les propriétaires terriens et leurs forces paramilitaires. Il me semblait qu'ils étaient en train d'inverser la violence symbolique qui, des générations durant, avaient rendu naturelle la domination des campesinos analphabètes à la peau sombre.

Passer du statut de créatures les plus méprisées sur terre (à savoir de travailleurs journaliers, sans terre ou presque, donnant des journées de travail obligatoires à des propriétaires omnipotents) à celui de guides de l'histoire: le peuple que la Bible prophétise. Les paysans se sentaient honorés de mourir pour leur cause parce qu'avant que cela ne se produise, ils avaient déjà été à moitié morts de souffrance ${ }^{15}$.

Mes notes de terrain, prises quelques jours avant l'invasion militaire en 1981, rapportent qu'un nombre étonnamment important de guérilleros salvadoriens s'étaient repentis d'avoir été pris par le passé dans des histoires d'alcoolisme ou de violence conjugale ${ }^{16}$. Dans la plupart des zones contrôlées par le FMLN, l'alcool était interdit et la violence domestique réprouvée. Indubitablement, ces formes de violence quotidienne avaient décru à mesure que le peuple s'organisait pour s'engager dans la violence politique. Dans un article publié au plus fort de la guerre, j'ai cité une phrase emblématique de l'un des guérilleros: "Nous étions des machistas. Nous avions l'habitude de boire beaucoup et de nous bagarrer. Mais alors l'Organisation nous a montré le chemin et nous avons canalisé cette violence pour le bien du peuple $»^{17}$. C'était effectivement passionnant de pouvoir rendre compte du sentiment de fierté qui émanait des paysans révolutionnaires. D'autres observateurs contemporains, comme Manlio Argueta, ont écrit avec éloquence sur le combat révolutionnaire des Salvadoriens, évoquant avec force la métamorphose des paysans salvadoriens. De victimes de la répression physique et de la violence symbolique lors des premières phases de répression de l'effervescence politique, ils s'étaient transformés en une armée pleine de dignité, composée de pauvres se battant activement pour leurs droits ${ }^{18}$. La politique néo-libérale de la représentation à El Barrio, USA

A la différence de la dynamique de libération que j'avais cru repérer derrière la violence politique au Salvador, la violence quotidienne qui envahissait les villes américaines, celle de la deuxième citation en exergue, m'était parue strictement oppressive et démobilisatrice. A la fin des années 1980, j'ai passé près de cinq ans avec ma famille dans un logement voisin d'une crackhouse (immeuble contrôlé par des dealers de crack) dans le quartier d'East Harlem à New York. Là, je me suis lié à un groupe de Portoricains, dealers de crack dans la rue, dont j'ai restitué les histoires de vie et observé les luttes quotidiennes pour continuer à survivre dans le respect de soi. Les fréquents passages à tabac, les agressions et les fusillades régulières entre les jeunes hommes avec lesquels je passais la plupart de mon temps, leurs mésententes continues avec leurs familles ont constitué des défis d'analyse théorique et politique bien plus 
éprouvants que la guerre au Salvador. La scène du commerce du crack ouvre une fenêtre sur les mécanismes de fusion et de conversion de la violence structurale et symbolique en violence quotidienne. Ségrégation extrême, inégalité sociale, misère matérielle s'expriment en premier lieu par les violences que les personnes socialement vulnérables s'infligent à elles-mêmes (via la consommation de drogues), imposent à leurs familles et amis (par la violence familiale ou le viol collectif entre adolescents), à leurs voisins et à la communauté (vols, cambriolages, attaques à main armée ou driveby shootings). Il en résulte une "culture de la terreur ${ }^{19}$ à l'échelle locale ou une violence quotidienne exacerbée qui met en place les frontières de ce que j'appelle l'apartheid urbain nord-américain ${ }^{20}$.

En tant que membre de la classe dominante des Etats-Unis, je m'inquiétais des implications politiques et même universitaires de mes descriptions des dealers de crack portoricains. Je craignais de contribuer à une "pornographie de la violence» qui submerge les explications structurales d'une décadence urbaine et se perd en sordides détails sur des agressions horribles et sanglantes. Comme l'a remarqué Laura Nader ${ }^{21}$, les études anthropologiques fondées sur l'observation participante parmi les plus démunis risquent d'avoir des effets d'humiliation publique qui se retournent contre eux. C'est particulièrement vrai dans le contexte de l'idéologie hégémonique néolibérale américaine qui, par définition, considère les pauvres comme moralement suspects. Toujours est-il que j'étais politiquement et théoriquement décidé à rendre entièrement compte de la souffrance sociale dans toutes ses facettes, en raison de l'extrême marginalité sociale et économique dans East Harlem. Ce dilemme m'a toutefois encouragé à me concentrer sur la violence structurale, et plus tard sur la violence symbolique ce qui, par définition, fait porter l'attention sur le niveau d'analyse macro des inégalités de pouvoir qui conditionnent la violence quotidienne.

A la fin de mon séjour à East Harlem, au moment même de la fin de la guerre froide, j'ai présenté un papier à une session de l'Association Américaine d'Anthropologie dans lequel je tentais de comparer les schémas et les expériences de violence dans les villages déchirés par la guerre du Salvador et dans les villes américaines en temps de paix $^{22}$. En soulignant la différence entre violence politique directe et violence structurale invisible, je croyais dépasser l'idéologie de la guerre froide que je ne faisais en fait que reproduire. Tout au long de mon analyse, je maintenais en effet une opposition morale entre la violence politique «utile» (worthy) qui rassemble les dominés contre la répression par un Etat autoritaire, et la violence «inutile » qui démobilise les personnes socialement vulnérables dans les sociétés démocratiques néolibérales. Mon souci de distinguer la bonne de la mauvaise violence, la violence de libération politique de la violence autodestructrice et irresponsable m'a rendu aveugle à la nature profondément mutilante de la violence politique en Amérique centrale. J'ai, en particulier, échoué à voir comment la répression et la résistance politiques en temps de guerre se reflètent dans une dynamique de violence quotidienne semblable à celle produite par la fusion de la violence structurale et de la violence symbolique en temps de paix.

$\mathrm{Au}$ lieu de cela, en m'inspirant de Gramsci, j'ai expliqué pourquoi l'expérience de la guérilla en termes de violence politique répressive au Salvador peut être interprétée comme une forme d'élévation de l'homme et de libération politique au travers de la douleur et de la colère qu'elle a engendrées. J'ai mis cette dynamique en opposition aux actes de violence quotidienne auxquels j'ai pu assister dans East Harlem et que j'avais alors interprétés comme l'expression d'une fausse conscience dans une société 
structuralement et symboliquement oppressive qui n'a plus besoin d'exercer de violence politique pour soutenir des structures d'inégalité. La théorie de Gramsci sur l'hégémonie est un outil de grande valeur, mais la façon dont j'avais catégorisé la violence comme «utile » ou « inutile » a modelé directement ce que j'étais à même de voir, d'entendre et de croire, ce que j'interprétais comme «données» et ce sur quoi je prenais mes notes de terrain, les questions que je jugeais pertinent de discuter et les débats dans lesquels je cherchais à m'engager. Au niveau empirique, alors que je m'étais amplement renseigné sur les types de souffrances causées par la violence structurale et symbolique dans une société socialement polarisée en temps de paix, j'ai simplifié à l'extrême et minimisé les ramifications de la terreur dans une société répressive déchirée par la guerre civile.

Réécrire les notes de terrain sur la guerre civile au Salvador

En me référant au texte cité en exergue, je peux encore parfaitement me remémorer cette nuit du 14 novembre 1981, quand je me suis retrouvé en train de courir au travers de la ligne de feu des militaires, avec un millier d'hommes, de femmes et d'enfants terrifiés. J'emploierais toutefois un vocabulaire différent aujourd'hui pour décrire les victimes. Maintenant, je pourrais par exemple me référer au " petit garçon » mutilé, se tordant de douleur sous mes yeux, avec son torse transpercé, comme à un «jeune combattant ", puisque, à peine âgé de quatorze ans, il portait une arme automatique. Les censures politiques de la guerre froide rendaient important, sinon impératif, de l'appeler «petit garçon » plutôt que "jeune combattant », car dans la vision martiale du conflit qui prévalait dans les années 1980, des adolescents qui portaient des armes automatiques méritaient d'être tués. Le pathos humain d'un enfant mourant dans un combat singulier en défendant sa famille contre des soldats gouvernementaux en maraude était à l'époque occulté.

De façon plus subtile, je garde en mémoire plusieurs versions de ces moments avant que je ne bute sur le corps de cet enfant soldat. J'ai réécrit des extraits ex-post-facto de mes notes de terrain, dix-huit ans après les faits, soulignant ce dont maintenant je me souviens. Lorsque j'avais préparé l'original de l'article en 1981, je n'avais pas été entièrement capable de me rappeler et d'analyser ces événements. Peut-être croyais-je que ces détails étaient sans importance. Encore une fois, dans le contexte de la guerre froide, mon premier souci était de mettre en lumière les vecteurs de pouvoir les plus contestables orientés contre les petits paysans du Salvador, c'est-à-dire, de fait, le régime militaire répressif maintenu par la politique étrangère américaine. J'ai évité de consigner ces souvenirs dans mes notes de terrain parce que je croyais qu'ils révéleraient un défaut de ma personnalité.

Quand les grenades ont touché le jeune combattant devant moi, j'ai plongé dans un fossé derrière des buissons. J'ai buté par accident sur une jeune mère qui se cachait déjà là où je m'étais précipité. Les yeux écarquillés, j'ai regardé son bébé de six mois environ. Il s'est mis à crier. A leur côté, j'étais essoufflé, grand, étranger, et puant d'une étrange odeur de transpiration et de peur : les pleurs du bébé se sont amplifiés en cris perçants et plaintifs.

La mère m'a soufflé à l'oreille « Vete ! Vete de aquí! Rápido ! " [Fichez le camp ! Vite !]. D'abord choqué, j'ai cru qu'elle m'en voulait et que par cruauté, elle me jetait sous la grêle des balles. Soudain, j'ai compris qu'elle tentait de sauver ma vie : les pleurs de son bébé commençaient à percer à travers le bruit des armes à feu. J'ai pris mes jambes à mon cou et me suis mis à courir droit devant moi, alors qu'une autre rafale de 
mitrailleuses s'abattaitau milieu des hurlements de la mère et du bébé juste derrière moi.

C'était ma première exposition en tant qu'observateur participant à cette sorte de trahison humaine que commettent les survivants dans les guerres contrerévolutionnaires : faire pleurer un bébé pour ensuite courir le plus loin possible dès lors qu'on réalise que ces cris peuvent attirer les balles. Cela m'a forcé à reconnaître la faillite de mon sens de la dignité humaine et de la masculinité et à remettre en question l'estime de moi-même. Cela confinait aussi à la violence symbolique en me mettant en colère tant contre moi que contre le FMLN qui faisait des civils les cibles de la répression gouvernementale.

Je ne suis pas certain que la mère et son bébé aient été tués par les balles attirées par les cris de l'enfant. Je crois qu'ils sont morts tous les deux. Si j'avais eu davantage de présence d'esprit, si je m'étais échappé un peu plus vite, lorsque sa mère me l'avait demandé, peut-être que ce bébé ne se serait pas mis à hurler et n'aurait pas attiré l'attention des soldats gouvernementaux. Une décennie plus tard, les conversations avec les guérilleros et leur famille m'ont appris que ces sentiments de culpabilité ou de trahison abondent dans les guerres contre-révolutionnaires. Ils sont inévitables pour survivre à la répression militaire et contribuent à une forme de violence symbolique, où les survivants concentrent leurs récriminations sur les victimes de leur camp ou sur leurs propres faiblesses de caractère, plutôt que sur les agents qui ont de fait imposé la terreur. Il en résulte souvent pour les témoins, qui s'en veulent d'avoir fait ce qu'ils ont dû faire pour survivre, une mise sous silence traumatisée de la brutalité des événements.

Durant cette même nuit où nous tentions d'échapper aux troupes gouvernementales qui nous encerclaient, j'ai dépassé des parents, des frères ou des sœurs un peu plus âgés, trébuchant sous le poids d'enfants terrifiés ou d'autres membres de la famille blessés. Alors que je fuyais, je me demandais si je devais m'arrêter et faire quelque chose pour eux. Convaincu que nous allions tous mourir, je courais pour rester en vie, mais je sentais bien que je trahissais ceux laissés à l'arrière. Alors que l'aube se levait, la plupart d'entre nous avons réussi à nous rassembler au fond d'un ravin pour nous $\mathrm{y}$ cacher. Nous espérions que les guérilleros pourraient nous assurer un peu de protection et nous priions pour que les hélicoptères du gouvernement ou les troupes au sol ratissant la zone ne nous trouvent pas. Quelques grenades bien placées ou quelques rafales de fusil automatique dirigées contre notre cachette auraient suffi à nous tuer. Par chance, lorsqu'un hélicoptère a survolé le ravin à seulement douze pieds au-dessus de nos têtes, il a mitraillé les combattants qui se trouvaient sur la pente de la colline et ne nous a pas détectés. Les membres de la guérilla, un peu au-dessus de nous, se sont dispersés rapidement et ont détourné avec succès le feu ennemi.

Après cette confrontation avec l'hélicoptère, je me suis retrouvé au beau milieu d'une famille qui tentait de calmer un bébé de dix-neuf jours dont la mère venait d'être tuée quelques heures plus tôt par une grenade alors qu'elle tentait d'échapper aux tirs. Ils m'ont demandé de les photographier, afin de garder une trace de leur histoire. Le nouveau-né avait été jeté à terre par le souffle de l'explosion, indemne, et avait été ramassé par sa tante dans le chaos. Les survivants de la famille n'avaient rien pour nourrir ce nouveau-né en pleurs, ils ne pouvaient que le bercer. Les huit jours suivants, le bon millier de personnes que nous étions est resté groupé, faisant les plus grands efforts pour étouffer nos bruits alors que nous changions de cachette et que nous 
récupérions un peu de nourriture dans les sous-bois. A plusieurs occasions, nous avons été localisés par les troupes gouvernementales, mitraillés par des hélicoptères de combat fournis par les Etats-Unis ou pourchassés par les troupes au sol. A chaque fois que nous décampions au plus vite pour nous cacher derrière des arbres ou des rochers, nous espérions que ceux qui portaient des armes allaient attirer l'ennemi dans une direction opposée.

Les jeunes, plus agiles et en bonne santé, avaient le plus de chances de survivre. Alors que le soleil se couchait, au huitième jour de notre fuite (douze jours après le début de l'attaque) et que nous étions pris sous des bombardements particulièrement intenses, je me suis mis à courir après un groupe de jeunes hommes qui semblaient bien connaître leur chemin. La plupart d'entre eux étaient des combattants qui avaient jeté les fusils avec lesquels ils étaient censés protéger leurs familles. Au lieu de quoi, nous avons couru toute la nuit, pris de ce que l'on peut appeler une terreur égoïste. De loin en loin, nous entendions encore les cris des enfants en pleurs qui attiraient les tirs. Nous avons échappé à la mort en nous réfugiant au Honduras voisin avant que le soleil ne se lève et tout le reste de la journée nous avons entendu les hélicoptères gouvernementaux frapper la masse lente et bruyante des civils que nous avions laissée derrière nous. Si mes camarades, depuis cette dernière nuit de fuite, ont survécu jusqu'à la fin de la guerre, il est vraisemblable qu'ils ne se sentent pas moins coupables aujourd'hui d'avoir survécu ${ }^{23}$.

Tout au long de la guerre, la propagande des gouvernements salvadorien et américain a dénoncé les guérilleros qui se cachaient au milieu des civils et qui par conséquent entraînaient la mort de ces derniers, pris dans des feux croisés. Le commandement même du FMLN était divisé sur sa politique visant à encourager - et parfois à exiger que les civils et les membres des familles des combattants restent sur les zones de combat. Les épouses étaient souvent en désaccord total sur cette question. Rétrospectivement, les mères ont parfois tenu leurs maris pour responsables de la mort de leurs enfants parce que les pères ont insisté pour qu'ils restent dans les villages afin de soutenir le FMLN. En 1983, un peu plus d'un an après cette opération de terre brûlée, les guérilleros ont changé de tactique et ont évacué la majorité des non-combattants hors des zones de guerre les plus chaudes. Il faut dire ici que la frontière entre " protecteur » et «lâche » est souvent ambiguë et n'a pas beaucoup de sens dans les guerres contre-révolutionnaires. Encore une fois, cet « espace liminal de la mort $»^{24}$, ou cette «zone grise $»^{25}$, atténue la responsabilité de ceux qui sont en premier lieu responsables de la terreur - à savoir dans ce cas, les militaires salvadoriens entrainés et soutenus par les Etats-Unis. Au contraire, les pièges de la violence symbolique - dans la confusion des sentiments de ne pas avoir été à la hauteur et d'être coupable d'une faute, accrue par les récriminations mutuelles - détournent l'attention de la violence politique répressive qui a créé les conditions de la terreur et imposé un douloureux choix entre la survie et la trahison.

Violence de guerre, violence de paix

Eté 1994. Alors que la guerre froide est terminée, je reviens dans les villages repeuplés par les combattants et les partisans de la guérilla, où j'avais été pris au piège lors de l'attaque de 1981. La chose la plus immédiatement tangible est la silencieuse brutalité de l'oppression économique. Mon premier ensemble de notes de terrain relatant cette visite décrit les cicatrices de la violence politique et structurale sur l'écologie locale et sur les corps des habitants : 
Juillet 1994

A cause de la rareté des terres, les villageois sont contraints de cultiver des terrains escarpés et rocheux. Comme s'il fallait ajouter les insultes aux blessures, les plaies mal soignées de la guerre rendent très difficile pour beaucoup de jeunes hommes de se rendre en boitant sur leursmilpas [lopins]ingrats. Même la terre paraît infirme et en colère : creusée par des ravines nées des fortes pluies et grêlée par des pierres saillantes et coupantes.

Tito, le fils de la femme chez laquelle nous vivons, s'est battu pendant près de dix ans avec le FMLN. Il boîte maintenant sur la pente de la colline où il tente de cultiver un peu de blé et de sorgho avec seulement une machette et un bâton pour bêcher. Il se sert de celui-ci comme d'une canne pour ne pas tomber dans son champ, mais grimace parfois quand il ressent les éclats d'obus encore insérés dans son mollet et dans son genou.

Personne n'est pourtant vraiment aimable avec Tito parce qu'il a des problèmes d'alcool maintenant. Mais il se dit surtout qu'il n'a pas été un combattant particulièrement brave pendant la guerre.

J'avais espéré que mon retour serait l'occasion d'une réunion cathartique entre moi et les gens avec lesquels je m'étais lié durant les quatorze jours du raid militaire de 1981. Cette expérience s'est révélée difficile, pleine d'illusions, un parcours sur la pointe des pieds dans un terrain miné de mauvaises actions, de déceptions et de trahisons. Mes amis ont insisté pour me raconter les erreurs militaires commises, les personnes blessées, abandonnées et laissées à l'ennemi. Pour me dire comment la vie d'un enfant particulièrement chétif et mentalement attardé avait été définitivement gâchée par l'absorption quotidienne de cinq pilules de valium administrées par sa mère pour calmer ses pleurs durant les combats, pour me dire encore quels combattants ont déserté. Ce que l'on ressent quand on tire dans la tête d'un ami blessé pour ne pas le laisser à l'ennemi et ne pas prendre le risque que, sous la torture, il révèle les identités et les positions des combattants de la guérilla. Ce que l'on ressent quand on a envoyé son fils de quatorze ans terrorisé rejoindre la guérilla, pour le voir tué à sa première sortie par un bombardement aérien. Treize ans après l'armistice, José, mon ami le plus proche, était encore préoccupé par le fait qu'il avait enterré plus de cent cinquante mines artisanales sur les chemins à flanc de coteau menant à son campement de guérilla. Il était persuadé que la plupart de ces mines avaient mutilé le pied d'un soldat, et que ses anciens ennemis, exactement comme lui et son père, claudiquaient sur les chemins abrupts des villages voisins, tentant d'accroître les récoltes de maïs pour maintenir leurs familles en vie.

Les notes prises au premier jour de mon enquête de terrain comprennent aussi une description de la coupure infectée sur le pied du frère de Tito, un petit garçon de dix ans. Cloué par la fièvre, il gémissait sans énergie dans un hamac installé dans la maison où je logeais. Il était impossible d'avoir accès à des soins médicaux dans la région. Je craignais que ce petit garçon ne meure du tétanos pour cette simple coupure. Mais il a survécu et en 1999, cinq ans plus tard, j'ai appris qu'il avait tué Tito, dont l'alcoolisme n'avait fait qu'empirer. Au procès en assises de son fils de quinze ans, la mère qui avait perdu son mari pendant la guerre civile à cause de la répression militaire implora le juge - sans succès - pour qu'il ne soit pas incarcéré, puisqu'il était le seul homme survivant de la maisonnée. Elle supplia le juge de le gracier, arguant que l'adolescent 
avait tenté de la protéger de son fils aîné qui la battait sauvagement quand il buvait trop.

$\mathrm{Au}$ cours de ce retour sur le terrain, une des histoires les plus choquantes que j'ai recueillies était celle d'une mère qui avait étouffé deux de ses jeunes enfants alors qu'elle les cachait dans une grotte au milieu d'une douzaine d'autres villageois. Ils ne nous avaient pas suivi la nuit où nous étions passés au travers des lignes gouvernementales. De crainte que les militaires salvadoriens ne détectent leur présence, les camarades avaient laissé le choix à la mère : soit elle quittait la grotte, soit elle bourrait d'un chiffon la bouche de ses enfants qui hurlaient de façon hystérique. Dix ans plus tard, la polémique avait toujours cours pour déterminer si le père avait eu raison par la suite de quitter sa femme pour avoir tué leurs deux enfants. Certains ont salué cet acte d'héroïsme d'une mère choisissant de sacrifier ses enfants pour tenter de sauver les vies de ses camarades cachés dans la grotte. Car il était évident que si elle avait quitté la caverne avec ses enfants en pleurs, elle aurait été capturée, et aurait pu sous la torture révéler la position de ses amis cachés. Des années plus tard, des doutes subsistaient pourtant toujours quant à la dignité morale de cette mère malchanceuse, troublant une fois encore la frontière entre l'héroïsme et la vilenie dans les guerres contre-révolutionnaires.

La question, trop douloureuse à poser, que j'introduisais de façon implicite dans la plupart de mes conversations lors de mon retour au Salvador, était de savoir si toute la souffrance et toute la violence de la guérilla n'avaient pas été vaines ${ }^{26}$. Poser cette seule question, dans le contexte d'une violence structurale toujours endurée par les anciens guérilleros et leurs familles, était ressenti comme une insulte. Leurs réponses rétrospectives, incertaines, parfois ambiguës sur la validité de leur combat impliquaient une autocritique : leur irresponsabilité et leur naïveté à se soumettre, eux et leurs familles, à la violence politique en soutenant le FMLN. Cette remise en question de l'utilité des sacrifices endurés a encouragé un isolement apolitique et une méfiance mutuelle. Toutefois, la majorité des ex-guérilleros et leurs familles continuent de soutenir les principaux idéaux de ce que maintenant ils appellent « la Guerre » plutôt que «la Révolution». Jusqu'à la fin des années 1990, ils ont constamment voté en faveur des anciens candidats FMLN, tant aux élections locales que nationales. En 2002, beaucoup d'anciens combattants et leurs familles solidaires ont alors commencé à réévaluer de façon bien plus positive leur engagement aux côtés du FMLN. Le fait qu'il n'y avait plus d'inquiétude sérieuse à propos des violations des droits de l'Homme était de plus en plus interprété comme la preuve de la victoire de la guérilla. Concrètement, d'un point de vue économique, ils reconnaissent s'en sortir plutôt mieux que les paysans qui ne se sont pas engagés du côté du FMLN. Aucun des petits paysans du village que j'ai étudié ne paie de loyer à un propriétaire, alors qu'avant la guerre civile, la très grande majorité versait des loyers exorbitants à un propriétaire, sous la forme à la fois de récoltes et de travail. Pratiquement, aujourd'hui, plus personne n'émigre durant la saison des moissons pour aller ramasser le coton ou le café, alors que dans la période précédant la guerre civile, la plupart des familles envoyaient régulièrement des hommes hors du village pour augmenter les ressources en travaillant sur les plantations destinées à l'exportation.

Les villageois se sont dits soulagés de ne plus avoir à quitter leurs familles chaque année pendant plusieurs mois. De manière plus subtile, ils étaient particulièrement fiers de ne plus avoir à subir les humiliations des anciens propriétaires de la région. Ils aiment à se rappeler combien les propriétaires étaient terrorisés durant la guerre civile 
et ils sont fiers qu'aucun d'eux n'ait osé revenir. Ceci est considéré comme une autre réalisation de la lutte du FMLN.

Pour finir sur une note contradictoire, il semble que durant la décennie suivant le processus de paix, les guérilleros ont émigré de façon disproportionnée vers les EtatsUnis, en tant que travailleurs journaliers sans papiers. Cela est peut-être dû au fait qu'ils sont plus enclins à prendre des risques pour améliorer leur avenir. Beaucoup font référence à l'argent obtenu en échange de la vente de leur arme automatique, grâce auquel ils ont payé un coyote [contrebandier passeur] pour franchir le Rio Grande. Par un coup de chance extraordinaire, dû à l'irrationalité de la politique d'immigration des Etats-Unis jusqu'à l'année 2001, ils ont été à même d'obtenir une carte de séjour en tant que réfugiés politiques fuyant la répression - un statut qui leur avait été refusé durant la guerre civile, au moment où ils en avaient réellement besoin. En effet, le statut de résident légal aux Etats-Unis est devenu, non sans ironie, l'une des plus grandes sources de profit économique pour les anciens combattants FMLN. L'économie du Salvador rural s'est progressivement axée autour de l'émigration clandestine aux Etats-Unis. Les versements d'argent des travailleurs aux Etats-Unis aux membres de leurs familles restés au Salvador ont représenté durant les années 1990 et au début des années 2000 une source de revenu plus importante que les exportations des produits agricoles $d u$ pays.

Violence mimétique

La sagesse apportée par le recul permet de voir clairement comment le mouvement révolutionnaire au Salvador a été traumatisé et corrompu par la violence contre laquelle il luttait. Par un processus quasi mimétique, la brutalité gouvernementale a été transposée dans les structures organisationnelles et dans les relations internes à la guérilla. La violence était devenue une banale nécessité instrumentale. Il y a plusieurs exemples bien connus de tueries au sein du commandement du FMLN. Le plus fameux est celui de Roque Dalton, le poète le plus célèbre du Salvador, tué par l'organisation de la guérilla dont il était membre depuis les années 1970. Il avait été accusé de "révisionnisme ", suite à un désaccord de stratégie politique quant à l'opportunité et l'utilité de s'engager dans la lutte armée ${ }^{27}$. Au milieu des années 1980, la femme qui était l'adjoint du commandant de la faction la plus importante de la coalition FMLN a été tuée dans une lutte sur la stratégie à poursuivre, continuer la lutte armée ou accepter la négociation. Elle aurait reçu soixante-huit coups de couteau du garde du corps de Cayetano Carpio, le chef de la faction, dont on croit savoir qu'il s'est suicidé au Nicaragua quelques mois plus tard, après que l'assassinat ait finalement été rendu public. La normalisation de la violence interne dans le contexte plus large de la violence politique prend sens si l'on pense la douleur et la terreur causées par la répression politique comme une "cocotte-minute " qui engendre de la violence quotidienne à travers une distorsion systématique des relations et des sensibilités sociales. Cela aide aussi à comprendre pourquoi le Salvador a eu pendant les années 1990, aprèsla fin de la guerre civile, le taux d'homicides par habitant le plus important du monde occidental. Dans la décennie suivant les accords de paix de la Saint Sylvestre 1991, il y a eu plus de victimes de la violence criminelle que pendant les dix années de guerre : 6250 morts par an pendant les années 1980 contre 8700 à 11000 durant les années $1990^{28}$.

En 2002, les ex-guérilleros et les supporters du FMLN étaient de plus en plus capables de parler de façon ouverte des assassinats commis dans leurs rangs. Certaines factions au sein du FMLN, comme les Fuerzas Populares de Liberación (FPL) et le Parti 
Communiste, ont été identifiées comme étant les plus enclines à pratiquer ce type d'assassinats ${ }^{29}$. D'une part, l'élite du FMLN en a débattu sous forme d'autocritique comme étant la conséquence d'un contrôle autoritaire vertical. D'autre part, les combattants de base ont aussi expliqué que les FPL étaient les plus portées à la suspicion parce que davantage infiltrées par les espions de l'armée que n'importe quelle autre faction, compte tenu de sa popularité. Les FPL étaient en effet la plus grande et la plus populaire des factions composant le FMLN. Elles recrutaient leurs membres rapidement et les envoyaient au combat sans trop enquêter sur eux et avec un entraînement minimal.

Le nombre des assassinats à l'intérieur des rangs du FMLN a varié de façon très importante d'une région à une autre, selon la personnalité des commandants locaux. Mayo Sibrian par exemple, dans la province de San Vincente, l'un des plus illustres commandants locaux des FPL est à présent accusé d'être le responsable de plusieurs centaines d'assassinats sommaires de combattants et de villageois en dépit de leur soutien. On dit aussi qu'il a pratiqué la torture sur ses propres troupes, selon des méthodes copiées sur celles de l'armée, alors qu'il était en proie à une psychose paranoïaque. On dit aussi qu'il a tué des femmes qui lui avaient refusé leurs faveurs sexuelles.

\section{NOTES}

1. TRADUCTION : CLOE RAYNA ET NICOLAS WUEST-FAMOSE, REVUE PAR DANIEL CEFAÏ. 2. . Galtung J., " Violence, Peace, and Peace Research », Journal of Peace Research, 6, 1969, pp. 167-191.

3. J. Galtung définit la violence structurale comme « la violence indirecte incorporée dans des ordres sociaux répressifs et créant d'énormes différences entre les potentialités et les réalités de l'auto-réalisation humaine ». Il affirme la spécificité de la violence structurale par rapport à la violence institutionnelle, insistant sur sa « nature plus abstraite (...) qui ne peut être attribuée à une institution précise ». La violence structurale est « naturelle comme l'air autour de nous... La formule générale derrière la violence structurale est l'inégalité, en particulier dans la distribution de pouvoir ». Voir Galtung J., « Peace : Research, Education, Action », Essays in Peace Research, Copenhagen, Christian Ejlers, 1975, pp. 173-175.

4. . Fanon F., The Wretched of the Earth, New York, Grove Press, 1963 [Les damnés de la terre, Paris, Maspéro, 1961].

5. . Camara H., Spiral of Violence, London, Sheed and Ward, 1971 ; Celam, Medellin Conclusions, Latin American Episcopal Conference, Bogotá, Celam, 1973 ; Martin-Baro, Ignacio, Writings for a Liberation Psychology, Boston, Harvard University Press, 1994. 6. . Farmer P., Infections and Inequalities : The Modern Plagues, Berkeley, University of California Press, 1999.

7. . Bourdieu P., Wacquant L., An Invitation to Reflexive Sociology, Chicago, University of Chicago Press, 1992, p. 162-173 et pp. 200-205 [Réponses. Pour une anthropologie réflexive, Paris, Seuil, 1992]. 
8. . Scheper-Hughes N., « Peace-Time Crimes », Social Identities, 1997, 3, pp. 471-497.

9. . Taussig M., Shamanism, Colonialism, and the Wild Man : A Study in Terror and Healing, Chicago, University of Chicago Press, 1987.

10. . Americas Watch, Managing the Facts : How the Administration Deals with Reports of Human Rights Abuses in El Salvador, New York, The Americas Watch Committee, 1985 ; United Nations, « From Madness to Hope : The 12-Year War in El Salvador. Report of the Commission on the Truth for El Salvador ", Document S/25500, 1993.

11. . Wallace S., « You Must Go Home Again : Deported L. A. Gangbangers Take Over El Salvador », Harper's Magazine, 2000, 301, 1803, pp. 47-56.

12. . Le bataillon conduisant cette offensive militaire sous le commandement du Colonel Sigfrido Ochoa incluait des membres de la Brigade Atlacalt entraînée par l'Armée des Etats-Unis. Selon un rapport de l'United Press International, « Ochoa a pris 15 journalistes le long d'un chemin recouvert de restes de vaches, de cochons et de chevaux à l'odeur infecte ». Cela a dû se passer le jour après que la plupart d'entre nous avions réussi à nous enfuir de cette zone jusqu'au Honduras. Ochoa a dit aux journalistes qu'il avait brûlé les cadavres de 250 guérilleros que ses troupes auraient tués pour « éviter une épidémie » («Afirman tropas del gobierno que mataron a 250 guerrillas y solo sufrieron 15 Bajas », Diario de las Americas, 20 novembre, 1981, p. 1). 13. . Sartre J.-P., « Preface », in Fanon F., op. cit., 1963, pp. 7-31.

14. . Bourgois P., « What U. S. Foreign Policy Faces in Rural El Salvador : An Eyewitness Account ", Monthly Review, 34(1), 1982, pp. 14-30.

15. . Bourgois P., idem, p. 24.

16. . Ceci faisait alors écho aux découvertes d'Oscar Lewis sur son terrain à Cuba, juste après la révolution de 1959 : "Les gens ont un nouveau sens de leur pouvoir et de leur importance. Ils ont reçu des armes et une doctrine qui glorifie la classe populaire comme espoir de l'humanité. (Un officiel cubain m'a dit qu'ils ont pratiquement éliminé la délinquance en donnant des armes aux délinquants !) » : Lewis O., "The Culture of Poverty ", in O. Lewis (ed.), Anthropological Essays, New York, Random House, 1970, pp. 67-80.

17. . Bourgois P., op. cit., 1982, pp. 24-25.

18. . Argueta M., Cuzcatlán : Where the Southern Sea Bends, New York, Vintage Books, 1987 [1986] ; Argueta M., Day of Life, New York, Vintage Books, 1983 [1980].

19. . Taussig M., op. cit.

20. . Bourgois P., In Search of Respect : Selling Crack in El Barrio, New York, Cambridge, 1995.

21. . Nader L., " Up the Anthropologist - Perspectives Gained from Studying Up », in D. Hymes (ed), Reinventing Anthropology, New York, Pantheon, 1972, pp. 284-311.

22. . Bourgois P., "The Pornography of Violence : Fieldwork in El Barrio and Beyond ", papier présenté à la 91ème réunion annuelle de l'American Anthropological Association, San Francisco, 2-6 décembre 1992.

23. . Cette interprétation de la violence symbolique dans des conditions extrêmes peut aider à comprendre le phénomène de la culpabilité des survivants parmi les victimes de l'holocauste. Cela peut aussi contribuer à expliquer le syndrome dit de Stockholm dans lequel les otages s'identifient avec la cause de leurs ravisseurs, comme dans le cas de Patty Hearst et de la Symbionese Liberation Army à San Francisco en 1974.

24. . Taussig M., op. cit.

25. . Levi P., The Drowned and the Saved, New York, Summit Books, 1986. 
26. . On notera la combinaison du point d'interrogation et du point d'exclamation dans le titre du volume édité par Ana Kelly Rivera recueillant les témoignages de femmes combattantes et de survivants de la répression militaire au Salvador : Valio la pena?! [Est-ce que ça valait la peine ? Ca valait la peine !] : Rivera A. K., Valió la pena ?! : testimonios de salvadorñas que vivieron guerra, San Salvador, El Salvador, Editorial Sombrero Azul, 1995.

27. . Joaquin Villalobos, un important commandant militaire du FMLN, est supposé être le responsable de l'assassinat de Roque Dalton. A la suite de l'armistice, Villalobos est devenu membre de l'Assemblée Nationale salvadorienne pendant une brève période et, au début des années 1990, il a formé des alliances stratégiques occasionnelles avec l'ARENA, le parti de droite qui représentait l'oligarchie dominante et qui avait des liens organiques avec les escadrons de la mort.

28. . De Cesare D., " The Children of War : Street Gangs in El Salvador ", NACLA, 1998, 32, 1, pp. 23-24 et Wallace S., op. cit., pp. 47-56.

29. . Gibb T., Untitled Book Manuscript, 399 pages.

INDEX

Mots-clés : guerre, violence

Index géographique : Amérique centrale

Index chronologique : post-guerre froide 